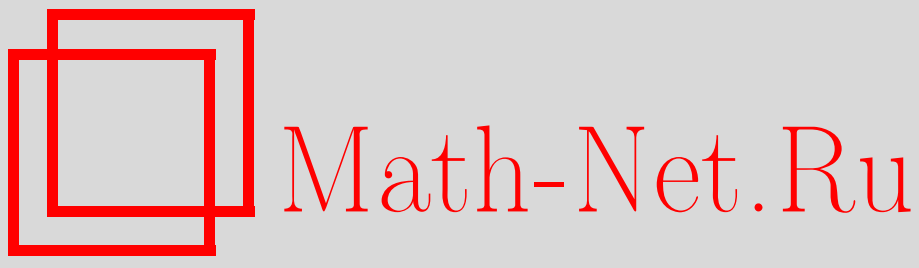

А. В. Горшков, О напряженно-деформированном состоянии цилиндра из упругопластического материала под действием давления в канале, Вестн. Сам. гос. техн. ун-та. Сер. Физ.-мат. науки, 2006, выпуск 42, 86-91

DOI: https://doi.org/10.14498/vsgtu416

Использование Общероссийского математического портала Math-Net.Ru подразумевает, что вы прочитали и согласны с пользовательским соглашением

http://www . mathnet.ru/rus/agreement

Параметры загрузки:

IP: 54.157 .27 .8

26 апреля 2023 г., 08:31:15

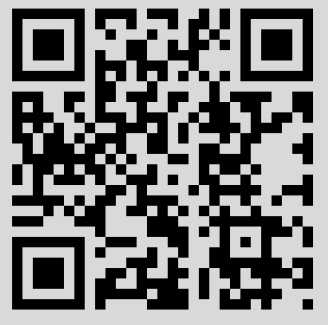




\section{О НАПРЯЖЕННО-ДЕФОРМИРОВАННОМ СОСТОЯНИИ ЦИЛИНДРА ИЗ УПРУГО-ПЛАСТИЧЕСКОГО МАТЕРИАЛА ПОД ДЕЙСТВИЕМ ДАВЛЕНИЯ В КАНАЛЕ}

С помощьью вариациионных принциипов исследуется напряженно деформированное состояние изилидра из упруго-вязкопластического материала при высоком давлении в канале. Эта модель описывает один из завершающих этапов пробоя циилндра высоковольтным электрическим разрядом. Определены напряжения в материале и скорость течения материала вблизи стенок канала.

При решении задач механики сплошной среды одна из важных проблем - получить конечномерные уравнения, адекватные непрерывным. Один из путей решения этой проблемы - использование вариационных принципов или законов сохранения. Если для непрерывной модели выполняется вариационный принцип или закон сохранения, то это свойство должно выполнятся и для дискретной модели. В качестве примера использования вариационного принципа приведено решение динамической осесимметричной задачи о напряженно деформированном со-

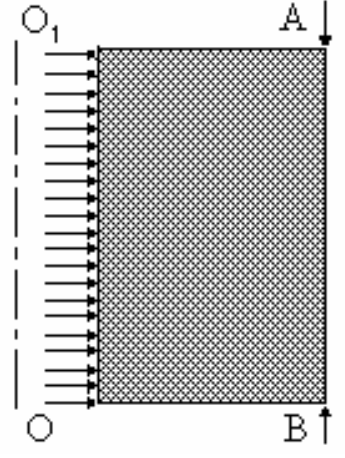

Р и с. 1. стоянии цилиндра из упруго-вязкопластического материала с тонким каналом по оси (рис. 1). Внешний радиус цилиндра $R_{0}=1$, радиус канала $R_{1}=0.1$, высота цилиндра $h=1$. В канале действует высокое быстро нарастающее давление $P(t)$. Внешняя поверхность свободная. Силой тяжести будем пренебрегать. На рис. 1 стрелками А и В отмечены точки закрепления, $O O_{1}$ - ось симметрии. Для решения используется вариационный принцип скоростей и напряжений, развитый в работах В.Л. Колмогорова $[1,2]$ в сочетании с методом конечных элементов [3].

Эта задача возникла при исследовании процесса разрушения образца электрическим разрядом. В процессе пробоя образуется тонкий канал, заполненный плазмой при высокой температуре и давлении. Важно определить динамические механические напряжения и скорость выброса материала из канала. Из-за высокой температуры и давления материал находится в пластическом состоянии. Задача упрощена по сравнению с исходной. Рассматривается только изотермический процесс. Однако и такая упрощенная схема позволяет определить скорость выброса материала из канала пробоя.

Используется Лагранжева начальная цилиндрическая система координат. Через $x^{i}$ будем обозначать текущие координаты точек среды, а через $y^{i}-$ их начальные значения, $i=1,2$. Ось $o y^{1}$ направлена по радиусу, ось $о y^{2}$ совпадает с осью цилиндра, ось $о y^{3}$ направлена по касательной,

$$
R_{1} \leq y^{1} \leq R_{0}, 0 \leq y^{2} \leq h .
$$

Осесимметричное движение в цилиндрической системе координат описывается уравнениями:

$$
\begin{aligned}
& \frac{\partial \sigma^{11}}{\partial y^{1}}+\frac{\partial \sigma^{12}}{\partial y^{2}}+\frac{\sigma^{11}}{y^{1}+u^{1}}-\sigma^{33}\left(y^{1}+u^{1}\right)=\rho_{0} \frac{\partial v^{1}}{\partial t}, \\
& \frac{\partial \sigma^{12}}{\partial y^{1}}+\frac{\partial \sigma^{22}}{\partial y^{2}}+\frac{\sigma^{12}}{y^{1}+u^{1}}=\rho_{0} \frac{\partial v^{2}}{\partial t} .
\end{aligned}
$$

Здесь $\sigma^{i j}$ - компоненты тензора напряжений, $v_{i}$ - компоненты вектора скорости, $u_{i}-$ компоненты вектора перемещений. С учетом осевой симметрии компоненты тензора скоростей деформаций $e_{i j}$ примут вид:

$$
e_{11}=\frac{\partial v^{1}}{\partial y^{1}}, \quad e_{12}=\frac{1}{2}\left(\frac{\partial v^{1}}{\partial y^{2}}+\frac{\partial v^{2}}{\partial y^{1}}\right), \quad e_{13}=0, e_{22}=\frac{\partial v^{2}}{\partial y^{2}}, \quad e_{23}=0, e_{33}=\left(y^{1}+u^{1}\right) v^{1}
$$


Материал цилиндра описывается следующими определяющими соотношениями: при $T<\sigma_{s}-$ $\sigma^{i j}=\lambda \varepsilon g^{i j}+2 \mu \varepsilon^{i j} ;$ при $T>\sigma_{s}$ материал вязкий идеально-пластический $-T=\sigma_{s}+\gamma H$. Объемная деформация остается упругой. Здесь $T=\sqrt{s_{i j} s^{i j} / 2}-$ интенсивность касательных напряжений, $s^{i j}=\sigma^{i j}-g^{i j} \sigma / 3-$ компоненты девиатора тензора напряжений, $\sigma-$ первый инвариант тензора напряжений,

$$
H=\left\{\frac{2}{3}\left[\left(e_{11}-e_{22}\right)^{2}+\left(e_{11}-e_{33}\right)^{2}+\left(e_{33}-e_{22}\right)^{2}\right]+e_{12}^{2}+e_{13}^{2}+e_{23}^{2}\right\}^{1 / 2}
$$

- интенсивность скоростей деформаций, $\sigma_{s}$ - предел текучести, $\varepsilon_{i j}$ - компоненты тензора деформации, $\varepsilon=\varepsilon_{i j} g^{i j}-$ первый инвариант тензора деформации, $g^{i j}-$ компоненты метрического тензора, в рассматриваемом случае $g^{i j}=0, \quad i \neq j, g^{11}=g^{22}=1, g^{33}=\left(y^{1}+u^{1}\right)^{-2}, \lambda, \mu$ - постоянные Ламе, $\gamma$ - коэффициент трения.

Так как материал цилиндра вязкопластический, то учитывается нелинейная связь перемещений и деформаций.

Функционал вариационного принципа имеет вид [1]:

$$
\delta\left\{I_{1}+I_{2}+I_{3}+I_{4}\right\}=0,
$$

где $I_{1}=\int_{V}\left[\int_{0}^{e_{i j}^{\circ}} s^{i j}(e) d e+\int_{0}^{s^{i^{\prime}}} e_{i j}(s) d s+\int_{0}^{e^{\prime}} \sigma(e) d e+\int_{0}^{\sigma^{\prime}} e(\sigma) d \sigma+\rho\left(w^{i}-g_{i}^{*}\right) v_{i}^{\prime}\right] d V ; I_{2}=-\int_{S_{f}} f_{*}^{i} v_{i}^{\prime} d S$;

$$
I_{3}=-\int_{S_{v}} f^{i} v_{i}^{*} d S ; I_{4}=-\int_{S_{S}}\left[f^{i} v_{i}^{*}-\int_{0}^{v_{s i}^{\prime}} f_{\tau}^{i}(v) d v-\int_{0}^{f_{\tau}^{i}} v_{s i}(f) d f\right] d S, e=e_{i j} g^{i j}=\frac{\partial v^{1}}{\partial y^{1}}+\frac{\partial v^{2}}{\partial y^{2}}+\frac{v^{1}}{y^{1}+u^{1}}
$$

- первый инвариант тензора скоростей деформации. На части поверхности тела $S_{f}$ заданы силы $f^{i}$, на $S_{v}$ - скорости, на $S_{s}$ - трение скольжения. Звездочкой отмечены заданные величины, штрихом - варьируемые.

В рассматриваемом случае поверхность $S_{f}$ состоит из двух участков:

1) поверхность канала, на которой задано давление $P(t)$ - известная функция времени;

2) поверхность торцов цилиндра, на которых давление равно давлению окружающей среды $P_{0} ; S_{v}$ - внешняя цилиндрическая поверхность, на которой скорость точек равна нулю. Тогда $I_{4}=0, I_{3}=0$,

$$
I_{2}=-2 \pi R_{1} P(t) \int_{0}^{h} v^{\prime 1} d y^{2}-2 \pi P_{0} \int_{R_{1}}^{R_{0}} y^{1} v^{\prime 2} d y^{1}
$$

Принцип скоростей и напряжений для изотропного тела в данном случае принимает вид

$$
\begin{gathered}
I=\int_{V}\left[\int_{0}^{\mathrm{H}} \mathrm{T}(\mathrm{H}) d \mathrm{H}+\int_{\tau_{s}}^{\mathrm{T}} \mathrm{H}(\mathrm{T}) d \mathrm{~T}+\int_{0}^{e^{\prime}} \sigma(e) d e+\int_{0}^{\sigma^{\prime}} e(\sigma) d \sigma+\rho w^{i} \mathrm{v}_{i}^{\prime}\right] d V- \\
-2 \pi R_{1} P(t) \int_{0}^{h} v_{1}^{\prime} d y^{2}-2 \pi P_{0} \int_{R_{1}}^{R_{0}} y^{1} v_{1}^{\prime} d y^{1}
\end{gathered}
$$

Для построения конечномерной модели используется метод конечных элементов. В области (1) вводится $N$ узлов с координатами $\left(y_{n}^{1}, y_{n}^{2}\right)$, которыми она разбивается на $M$ треугольных 
элементов с вершинами в заданных узлах. Так как задача осесимметричная, то конечные элементы - фактически трехмерные тела вращения. На каждом элементе вводится система локальных координат и локальная нумерация. В качестве первой выбирается вершина, ближайшая к оси симметрии и имеющая наименьшие координаты $y^{1}$ и $y^{2}$. Остальные нумеруются обходом против часовой стрелки. Первая вершина выбирается за начало координат, ось $o z^{1}$ совпадает со стороной, проходящей через вершины 1,2 . Ось $o z^{2}$ перпендикулярна $o z^{1}$.

На элементе задается система локальных базисных функций

$$
\varphi_{i}=a_{i} y^{1}+b_{i} y^{2}+c_{i}, i=1,2,3
$$

Коэффициенты $a_{i}, b_{i}, c_{i}$ зависят от координат вершин элемента и выбираются так, чтобы система функций $\varphi_{i}$ была нормализованной. В локальной системе координат базисные функции имеют вид

$$
\varphi_{1}\left(z^{1}, z^{2}\right)=-\frac{z^{1}}{l}-\frac{z^{2}(l-p)}{l q}+1, \quad \varphi_{2}\left(z^{1}, z^{2}\right)=\frac{z^{1}}{l}-\frac{z^{2} p}{l q}, \quad \varphi_{3}\left(z^{1}, z^{2}\right)=-\frac{z^{2}}{q},
$$

где $(l, 0)$ - координаты второго узла элемента в локальной системе, $(p, q)$ - координаты третьего узла, $q>0$ - по построению. При этом коэффициенты разложения искомых функций по базисным равны значениям неизвестных функций в узлах сетки.

Поле перемещений на элементе аппроксимируется локальными базисными функциями:

$$
u^{(m) i} \approx u^{(m) n i} \varphi_{n}^{(m)}(\stackrel{I}{y})
$$

Здесь $m$ - номер элемента, $n$ - номер функции, $i$ - номер компоненты вектора перемещений. Аналогично строится поле скоростей точек среды. Здесь и далее используется правило неявного суммирования по повторяющимся индексам. По индексу, взятому в скобки, суммирование должно быть указано явно.

Соответственно, глобальные перемещения аппроксимируются выражениями:

$$
U^{i} \approx \sum_{m} \Lambda_{k}^{n} \varphi_{n}^{(m)}(y) u^{k i}=\Phi_{k}(\stackrel{r}{y}) U^{k i}
$$

$U^{i}-i$-ая компонента вектора перемещения, $U^{k i}-i$-ая компонента перемещения узла с глобальным номером $k, \Phi_{k}(\underset{r}{y})=\sum_{m=1}^{M} \stackrel{(m)}{\Lambda_{k}^{n}} \varphi_{n}^{(m)}\left(\stackrel{r}{y)}\right.$ - глобальная базисная функция. Таблица $\Lambda_{k}^{n}$ задает соответствие глобальных номеров узлов сетки и локальных номеров узлов элементов:

$$
\Lambda_{n}^{k}= \begin{cases}1, & \text { если глобальный узел } k \text { связанной модели совпадает с узлом } n \text { элемента } m ; \\ 0, & \text { в противном случае. }\end{cases}
$$

Аналогично аппроксимируются скорости и ускорения:

$$
V^{i} \approx \sum_{m} \Lambda_{k}^{n} \varphi_{n}^{(m)}(y) v^{k i}=\Phi_{k}(y) V^{k i}, W^{i} \approx \sum_{m} \Lambda_{k}^{n} \varphi_{n}^{(m)}(y) w^{k i}=\Phi_{k}(y) W^{k i} .
$$

Обратная процедура - разбиение области на конечные элементы, выполняется с помощью

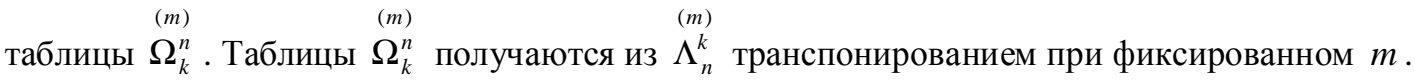

После подстановки конечно-элементных представлений в (3) и интегрирования, последнее слагаемое в скобке примет вид 


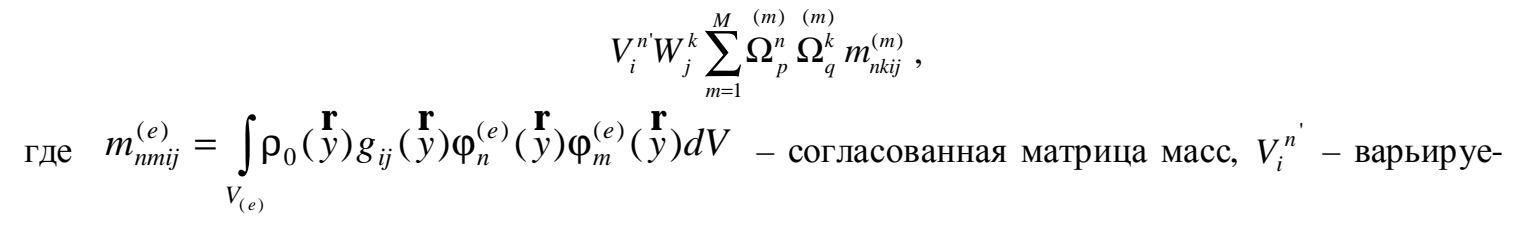
мая $i$-ая компонента скорости в узле $n, W_{j}^{k}-j$-ая компонента ускорения в узле $k$. Сумму

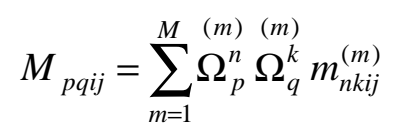

назовем глобальной обобщенной матрицей масс. В рассматриваемом случае таблица разобьется на три независимых матрицы, причем третья будет представлять моменты инерции элементов относительно оси симметрии. Так как задача осесимметричная, то третья таблица существенной роли не играет.

Аппроксимация скоростей деформаций на элементе имеет вид

$$
e_{i j}^{(m)}=\frac{1}{2} \Omega_{n}^{k}\left(V_{i}^{n} \frac{\partial \varphi_{k}^{(m)}}{\partial y_{j}}+V_{j}^{n} \frac{\partial \varphi_{k}^{(m)}}{\partial y_{i}}\right) .
$$

Из полученных выражений видно, что на элементе скорость деформаций не зависит от координат, и вариация первого слагаемого в скобке в (3) по скоростям имеет вид

$$
\begin{gathered}
\delta \int_{V}^{H_{0}^{\prime}} T(H) d H d V=\sum_{m=1}^{M}\left(\sigma_{s}+\gamma H^{(m)}\right) \delta H^{(m)} S_{(m)}, \\
\delta H^{(m)}=\sum_{i, j, k, p} \Omega_{n}^{k} \frac{\partial H^{(m)}}{\partial e_{i j}^{(m)}} \frac{\partial e_{i j}}{\partial V_{p}^{n}} \delta V_{p}^{n}, \frac{\partial H^{(m)}}{\partial e_{i i}^{(m)}}=\frac{4 e_{i i}^{(m)}}{3 H^{(m)}}, \quad \frac{\partial H^{(m)}}{\partial e_{i j}^{(m)}}=\frac{e_{i j}^{(m)}}{H^{(m)}}, i \neq j, \\
\frac{\partial e_{i j}^{(m)}}{\partial V_{p}^{n}}= \begin{cases}0, \quad p \neq i, p \neq j, \\
\frac{\partial \varphi_{k}^{(m)}}{\partial y_{i}}, & p=i, \\
\frac{\partial \varphi_{k}^{(m)}}{\partial y_{j}}, & p=j,\end{cases}
\end{gathered}
$$

где $S_{(m)}$ - площадь элемента $m, H^{(m)}$ - интенсивность скоростей деформаций на элементе $m$, $V_{p}^{n}-n$ компонента вектора скорости в узле с глобальным номером $p$. Функцию $1 / H^{(m)}$ будем аппроксимировать выражением $\frac{1}{H^{(m)}} \approx \Phi_{k} \frac{1}{H^{(m) k}}$.

Аналогично строится вариация интенсивности напряжений $T$.

В результате варьирования представленных выражений по узловым скоростям получим систему обыкновенных дифференциальных уравнений вида

$$
\begin{aligned}
& \frac{d U_{n}^{i}}{d t}=V_{n}^{i}, \\
& M_{n k i j} \frac{d V_{k}^{i}}{d t}=f_{n}^{i}(t, U, V),
\end{aligned}
$$

которая решается численно. Здесь $i$ - номер компоненты вектора перемещений или скорости, $k-$ номер глобального узла. Используется неявная разностная схема:

$$
\begin{aligned}
& U_{i, j+1}^{n}-U_{i, j}^{n}=\frac{\Delta t}{2}\left(V_{i, j+1}^{n}+V_{i, j}^{n}\right), \\
& M_{n k i l}\left(V_{l, j+1}^{k}-V_{l, j}^{k}\right)=\frac{\Delta t}{2}\left(f_{i}^{n}\left(t_{j+1}, U_{j+1}, V_{j+1}\right)+f_{i}^{n}\left(t_{j}, U_{j}, V_{j}\right)\right) .
\end{aligned}
$$

Индекс $j$ - номер временного слоя; $U_{j}$ показывает, что функция может зависеть от значений перемещений и скорости во всех узлах сетки для данного временного слоя. Эта схема для ли- 
нейной механической задачи обеспечивает сохранение полной энергии системы на шаге времени.

На рис. 2 и 3 представлены графики изменения интенсивности скорости сдвига в среднем сечении цилиндра $y^{2}=0.5$ и интенсивности касательных напряжений. Из графиков видно, что вдоль радиуса распространяется волна сдвига. Расчеты проводились для цилиндра с размерами $R_{0}=1, R_{1}=0.1, h=1$; модуль упругости $E=10^{11}$; коэффициент Пуассона $v=0.3 ; \sigma_{s}=10^{7}$; скорость роста давления $d P / d t=5 \cdot 10^{7}$.

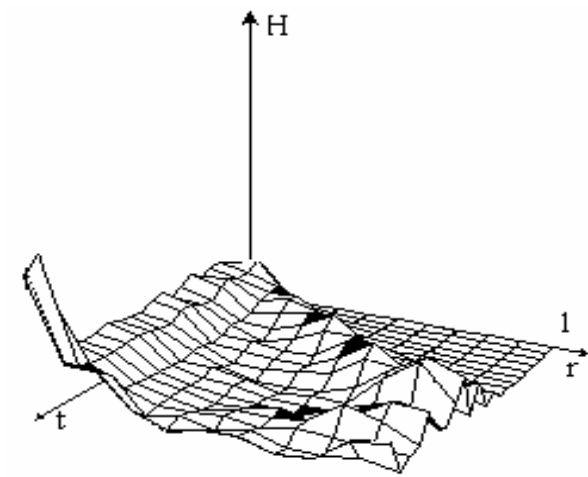

Р и с. 2.

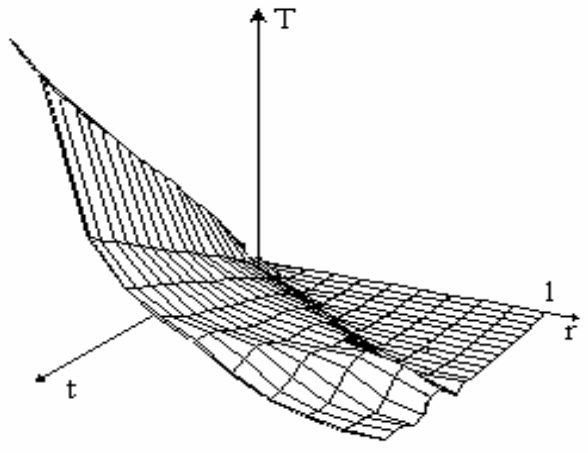

Р и с. 3.

На рис. 4 и 5 представлены профили интенсивности касательных напряжений для моментов времени $t=0.0003$ и $t=0.0007$ Увеличение напряжений в точках с координатами $h=0, r=1$. и $h=1, r=1$ на рис. 5 объясняется тем, что это точки закрепления.

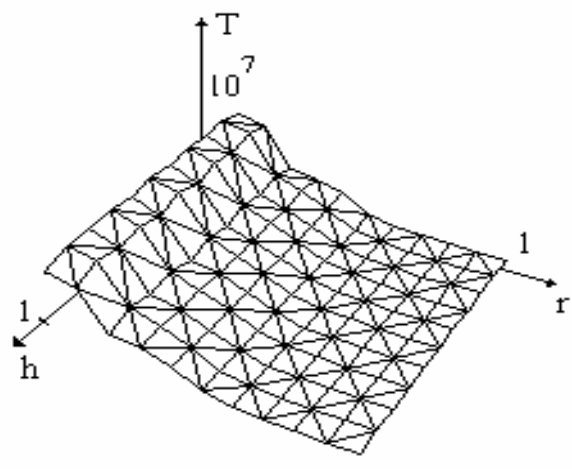

$\mathrm{t}=0.0003$

Р и с. 4.

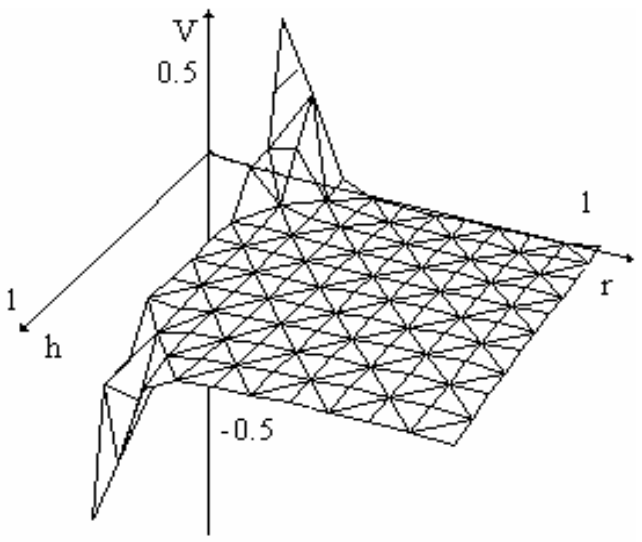

Р и с. 6.

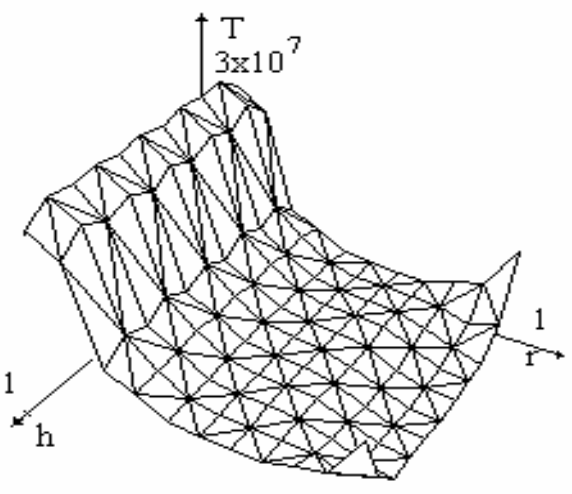

$\mathrm{t}=0.0007$

Р и с. 5.

Предложенный алгоритм позволяет определить скорость выброса материала, график распределения которой представлен на рис. 6.

Рассматривалось несколько вариантов сетки. Первый - сетка равномерная по радиусу и сетка с увеличивающимся шагом по радиусу. Сравнивались также сетки с различным разбиением области на элементы. Сравнение результатов показало, что наилучшие результаты обеспечивает равномерная сетка. Менялось также расположение треугольников. Наилучший вариант соответствует симметричному расположению. 


\section{БИБЛИОГРАФИЧЕСКИЙ СПИСОК}

1. Колмогоров В.Л. Вериинин С.В., Спевак Л.Ф., Гориков А.В. Применение метода расчета напряженно- деформированного состояния для некоторых задач обработки металлов давлением // Тез. докл. 10-й зимней школы по механике сплошных сред, Пермь, 1995.

2. Колмогоров В.Л., Федотов В.П., Горшков А.В. Трехмерный анализ напряженно-деформированного состояния // Ковочно-штамповочное производство. №8. 1998. С.23-28

3. Оден Дж. Конечные элементы в нелинейной механике сплошных сред. М.: Мир, 1976. 466 с.

Работа выполнена при поддержке РФФИ, проект № 04-01-00274.

Поступила 26.12.2005 2. 